\title{
FATOU AND KORÁNYI-VÁGI TYPE THEOREMS ON THE MINIMAL BALL
}

\author{
NGUYÊN VIÊT ANH
}

Abstract

In this paper we develop the $H^{p}(p \geq 1)$ theory on the minimal ball. After identifying the admissible approach regions, we establish theorems of Fatou and Korányi-Vági type on this ball.

\section{Introduction and statement of our main results}

It is well-known from the work of Stein [17] that holomorphic functions of $H^{p}$ class on a bounded domain in $\mathbb{C}^{n}$ with $\mathcal{C}^{2}$-boundary converge almost everywhere to their boundary values, provided the limit is taken inside certain natural approach regions. Boundary behavior of $H^{p}$ functions on smooth domains is rather well understood, see for example [6], $[\mathbf{9}],[\mathbf{1 0}],[\mathbf{1 1}]$, etc.

In this paper we are interested in Fatou type theorems for $H^{p}(p \geq 1)$ functions and Korányi-Vági type theorems on a non piecewise smooth domain: The minimal ball $\mathbb{B}_{*}$. This is the convex circular domain defined for $n \geq 2$ by

$$
\mathbb{B}_{*}:=\left\{z \in \mathbb{C}^{n}:|z|^{2}+|z \bullet z|<1\right\},
$$

where $z \bullet w:=\sum_{j=1}^{n} z_{j} w_{j}$. This is the unit ball with respect to the norm

$$
N_{*}(z):=\sqrt{|z|^{2}+|z \bullet z|}, \quad z \in \mathbb{C}^{n} .
$$

The norm $N:=\frac{N_{*}}{\sqrt{2}}$ was introduced by Hahn and Pflug [4], and was shown to be the smallest complex norm in $\mathbb{C}^{n}$ with the following properties $N(x)=|x|$ for $x \in \mathbb{R}^{n}$ and $N(z) \leq|z|$ for $z \in \mathbb{C}^{n}$.

Set $V:=\left\{z \in \mathbb{C}^{n} \backslash\{0\}: z \bullet z=0\right\}$. The singular part of the boundary of $\mathbb{B}_{*}$ is obviously the set $\partial \mathbb{B}_{*} \cap V$. The regular part $\partial \mathbb{B}_{*} \backslash V$ consists of all strictly pseudoconvex points. Moreover $\mathbb{B}_{*}$ is neither homogeneous nor Reinhardt (see [5], [13]). Function theory on the minimal ball was

2000 Mathematics Subject Classification. Primary 32A40, 32A35.

Key words. Admissible approach regions, Fatou type theorem, Korányi-Vági type theorem, the minimal ball. 
studied by several authors (see $[\mathbf{1 2}],[\mathbf{1 4}],[\mathbf{1 5}],[\mathbf{8}],[\mathbf{7}],[\mathbf{1 8}]$ ). In his recent work $[\mathbf{1 9}]$, E. H. Youssfi developed a method for computing the Bergman and Szegö kernel of a new class of pseudoconvex domains including the minimal ball. His paper is the main motivation for the present work. As in $[\mathbf{8}],[\mathbf{1 9}]$, our method consists of two steps. At the first step we study the problem on an auxiliary complex manifold $\mathbb{M}$. At the second step we transplant the results obtained on the complex manifold $\mathbb{M}$ to $\mathbb{B}_{*}$ by means of a proper holomorphic mapping.

This paper is organised as follows: In the first section we define the admissible approach regions for the minimal ball $\mathbb{B}_{*}$ and state our main results.

In Section 2 we give some properties of the system of admissble approach regions.

In Section 3 we discuss theorems of Fatou type and Korányi-Vági type on the complex manifold $\mathbb{M}$. We next transplant these results to $\mathbb{B}_{*}$ in Section 4 in order to prove our main Theorems A, B and C below.

We now identify the admissible approach regions.

Definition 1.1. For $\alpha>1$ and $\zeta \in \partial \mathbb{B}_{*}$, we let the "admissible approach region" $D_{\alpha}^{*}(\zeta)$ be the set of all $z \in \mathbb{B}_{*}$ such that

$$
\min _{\epsilon \in\{-1,1\}}|1-\langle z, \zeta\rangle-\epsilon \sqrt{(z \bullet z) \overline{(\zeta \bullet \zeta)}}|<\frac{\alpha}{2}\left(1-N_{*}^{2}(z)\right)
$$

where $\langle.,$.$\rangle denotes the standard Hermitian inner product.$

Definition 1.2. A function $f$ defined on $\mathbb{B}_{*}$ is said to have admissible limit $\lambda$ at a point $\zeta \in \partial \mathbb{B}_{*}$ if $f$ converges to $\lambda$ along $D_{\alpha}^{*}(\zeta)$ for every $\alpha>1$.

The question naturally arises how the system of admissible approach regions is different from the system of approach regions defined by E. Stein (see [17, p. 32]). Part (i) of Proposition 2.1 below asserts that on any compact set in the regular part of $\partial \mathbb{B}_{*}$, these two systems are, in a sense, equivalent to each other. Furthermore, part (ii) of that proposition says that the admissible approach regions are tangential to the regular part $\partial \mathbb{B}_{*} \backslash V$ in the complex tangential directions. Note that the Korányi approach regions for the Euclidean balls of $\mathbb{C}^{n}$ also have this geometric property. 
In order to state our main results we need some notations. Let $\mu$ be a positive Borel measure on $\partial \mathbb{B}_{*}$ and $p>0$. Then the Hardy space $H^{p}\left(\mathbb{B}_{*}, \mu\right)$ is defined by

$$
H^{p}\left(\mathbb{B}_{*}, \mu\right):=\left\{f \in H\left(\mathbb{B}_{*}\right),\|f\|_{H^{p}\left(\mathbb{B}_{*}, \mu\right)}^{p}:=\sup _{0<r<1} \int_{\partial \mathbb{B}_{*}}\left|f_{r}\right|^{p} d \mu<\infty\right\},
$$

where $f_{r}$ denotes the dilated function defined for $N_{*}(z)<\frac{1}{r}$ by $f_{r}(z):=$ $f(r z)$.

Let $\theta$ be the Lebesgue surface measure of $\partial \mathbb{B}_{*} \backslash V$. Throughout the paper, $\mathcal{S}_{\mathbb{B}_{*}}$ and $\mathcal{P}_{\mathbb{B}_{*}}$ denote the Szegö and Poisson-Szegö projection of $\mathbb{B}_{*}$ (with respect to the measure $\theta$ ) respectively. The letter $C$ will denote a finite constant that is not necessarily the same at each occurence.

Our first main result is the following

Theorem A. If $f \in H^{p}\left(\mathbb{B}_{*},|\zeta \bullet \zeta|^{s} d \theta\right), p \geq 1$ and $s \in \mathbb{R}$,

(i) then $f$ has finite admissible limits $f^{*}$ a.e. $[\theta]$ on $\partial \mathbb{B}_{*}$ and $f^{*} \in$ $L^{p}\left(\partial \mathbb{B}_{*},|\zeta \bullet \zeta|^{s} d \theta\right)$

(ii) if moreover $p>1$ and $-2<s<2 p-2$, then $f=\mathcal{S}_{\mathbb{B}_{*}}\left[f^{*}\right]=\mathcal{P}_{\mathbb{B}_{*}}\left[f^{*}\right]$.

Theorem A (or Fatou type theorem for the minimal ball) is only proved here for $p \geq 1$. It seems to be of interest to know whether part (i) of this theorem holds for all $p>0$. The Hardy spaces $H^{p}\left(\mathbb{B}_{*},|\zeta \bullet \zeta|^{\frac{p-2}{2}} d \theta\right)$ appear naturally in studying the $H^{p}$ theory associated to the minimal ball (see the works $[\mathbf{8}],[\mathbf{7}],[\mathbf{1 9}]$ ).

Next, if $u \in \mathcal{C}\left(\mathbb{B}_{*}\right)$ and $\alpha>1$, the maximal function $M_{\alpha} u: \partial \mathbb{B}_{*} \rightarrow$ $[0, \infty]$ is defined by

$$
\left(M_{\alpha} u\right)(\zeta):=\sup \left\{|u(z)|: z \in D_{\alpha}^{*}(\zeta)\right\} .
$$

We now state the second main result.

Theorem B. If $1<p<\infty$ and $-2<s<\infty$, then for every $f \in$ $H^{p}\left(\mathbb{B}_{*},|\zeta \bullet \zeta|^{s} d \theta\right)$,

(i) $\int_{\partial \mathbb{B}_{*}}\left|\left(M_{\alpha} f\right)(\zeta)\right|^{p}|\zeta \bullet \zeta|^{s} d \theta(\zeta) \leq C(s, \alpha, p) \int_{\partial \mathbb{B}_{*}}\left|f^{*}(\zeta)\right|^{p}|\zeta \bullet \zeta|^{s} d \theta(\zeta)$,

(ii) $\lim _{r \rightarrow 1^{-}}\left\|f^{*}-f_{r}\right\|_{L^{p}\left(\partial \mathbb{B}_{*},|\zeta \bullet \zeta|^{s} d \theta\right)}=0$

$$
\text { and }\|f\|_{H^{p}\left(\mathbb{B}_{*},|\zeta \bullet \zeta|^{s} d \theta\right)}=\left\|f^{*}\right\|_{L^{p}\left(\partial \mathbb{B}_{*},|\zeta \bullet \zeta|^{s} d \theta\right)} .
$$

Theorem B should be compared with the analogous results (Theorems 5.6.5 and 5.6.6 of [16]) in the case of the Euclidean unit ball.

Finally our third main result is the following 
Theorem C. Let $1<p<\infty$ and $\alpha>1$. If $\frac{p}{2}-2<s<\frac{3 p}{2}-2$, then there exists $C(s, \alpha, p)<\infty$ such that

$$
\int_{\partial \mathbb{B}_{*}}\left|M_{\alpha} \mathcal{S}_{\mathbb{B}_{*}}[f](\zeta)\right|^{p}|\zeta \bullet \zeta|^{s} d \theta(\zeta) \leq C(s, \alpha, p) \int_{\partial \mathbb{B}_{*}}|f(\zeta)|^{p}|\zeta \bullet \zeta|^{s} d \theta(\zeta)
$$

for all $f \in L^{p}\left(\partial \mathbb{B}_{*},|\zeta \bullet \zeta|^{s} d \theta\right)$.

We note that an analogue of Theorem $\mathrm{C}$ in the case of the Euclidean unit ball is the classical Korányi-Vági theorem (Theorem 6.3.1 of [16]).

\section{Some properties of the system of admissible approach regions}

We have the following

Proposition 2.1. (i) For $\alpha>1$ and $\zeta \in \partial \mathbb{B}_{*} \backslash V$, let $\mathcal{A}_{\alpha}(\zeta)$ be the classical approach region $[\mathbf{1 7}$, p. 32] defined by

(2.1) $\mathcal{A}_{\alpha}(\zeta):=\left\{z \in \mathbb{B}_{*}:\left|\left\langle z-\zeta, \nu_{\zeta}\right\rangle\right|<\alpha \delta(z),|z-\zeta|^{2}<(\alpha-1) \delta(z)\right\}$.

Here $\nu_{\zeta}$ denotes the unit outward normal at $\zeta$ and $\delta(z)$ is the distance from $z$ to $\partial \mathbb{B}_{*}$. (Notice that since $\mathbb{B}_{*}$ is convex, $\delta(z)$ is smaller than the distance from $z$ to the tangent space at $\zeta$.) Let $K$ be a compact in $\partial \mathbb{B}_{*} \backslash V$. Then there exist $\beta, \gamma>1$ such that

$$
\mathcal{A}_{\alpha}(\zeta) \subset D_{\beta}^{*}(\zeta) \quad \text { and } \quad D_{\alpha}^{*}(\zeta) \subset \mathcal{A}_{\gamma}(\zeta), \quad \forall \zeta \in K \text {. }
$$

(ii) For $\alpha>1$ and $\zeta \in \partial \mathbb{B}_{*} \backslash V$, the admissible approach region $D_{\alpha}^{*}(\zeta)$ is tangential to $\partial \mathbb{B}_{*} \backslash V$ in the direction of $T_{\zeta}^{\mathbb{C}}\left(\partial \mathbb{B}_{*} \backslash V\right)$.

Proof: The proof of assertion (ii) is postponed until Section 4. Here we only prove assertion (i).

A little calculation gives that

(2.3) $\nu_{\zeta}=\frac{1}{\sqrt{2}}\left(\zeta_{1}+\overline{\zeta_{1}} \frac{\zeta \bullet \zeta}{|\zeta \bullet \zeta|}, \ldots, \zeta_{n}+\overline{\zeta_{n}} \frac{\zeta \bullet \zeta}{|\zeta \bullet \zeta|}\right) \quad$ and $\quad\left\langle\zeta, \nu_{\zeta}\right\rangle=\frac{1}{\sqrt{2}}$.

For $z \in \mathbb{B}_{*}$, let $w \in \partial \mathbb{B}_{*}$ such that $\|z-w\|=\delta(z)$. A geometric argument shows that $w \in \partial \mathbb{B}_{*} \backslash V$ and $w-z=\delta(z) \nu_{w}$. This, combined with (2.3), implies

$$
z_{k}=w_{k}-\frac{\delta(z)}{\sqrt{2}}\left(w_{k}+\overline{w_{k}} \frac{w \bullet w}{|w \bullet w|}\right), \quad \forall 1 \leq k \leq n .
$$

Next, substituting the latter equation into the expression of $N_{*}^{2}(z)$ and using the equality $N_{*}^{2}(w)=1$, we obtain, after some simplifications,

$$
1-N_{*}^{2}(z)=\min \{2|w \bullet w|, \sqrt{2} \delta(z)(2-\sqrt{2} \delta(z))\} .
$$


Now we prove the first assertion in (2.2). Consider $\zeta \in K$ and $z \in \mathcal{A}_{\alpha}(\zeta)$. By (2.1) and (2.3), we get

$$
\left|1-\sum_{k=1}^{n} z_{k}\left(\overline{\zeta_{k}}+\zeta_{k} \frac{\overline{\zeta \bullet \zeta}}{|\zeta \bullet \zeta|}\right)\right|<\alpha \sqrt{2} \delta(z) .
$$

Thus for $z$ close enough to $\zeta$,

$$
\begin{aligned}
|1-\langle z, \zeta\rangle-\sqrt{(z \bullet z) \overline{(\zeta \bullet \zeta)}}| & <\left|1-\sum_{k=1}^{n} z_{k}\left(\overline{\zeta_{k}}+\zeta_{k} \frac{\overline{\zeta \bullet \zeta}}{|\zeta \bullet \zeta|}\right)\right| \\
& +\left|\sqrt{(z \bullet z) \overline{(\zeta \bullet \zeta)}}-\sum_{k=1}^{n} z_{k} \zeta_{k} \frac{\overline{\zeta \bullet \zeta}}{|\zeta \bullet \zeta|}\right|=I+I I .
\end{aligned}
$$

From (2.5), we have $I<\alpha \sqrt{2} \delta(z)$.

We now estimate $I I$ :

$$
\begin{aligned}
I I & =\left|\sqrt{\overline{\zeta \bullet \zeta}}(\sqrt{z \bullet z}-\sqrt{\zeta \bullet \zeta})-\sqrt{\overline{\zeta \bullet \zeta}} \cdot \sum_{k=1}^{n}\left(z_{k}-\zeta_{k}\right) \frac{\partial \sqrt{z \bullet z}}{\partial z_{k}}\right|_{z=\zeta} \mid \\
& =O\left(|z-\zeta|^{2}\right)<\sqrt{2} M(\alpha-1) \delta(z),
\end{aligned}
$$

where the last inequality follows from (2.1) and the assumption that $z \in \mathcal{A}_{\alpha}(\zeta), M$ is a constant that depends only on $K$.

Suppose $z$ is close enough to the boundary of $\mathbb{B}_{*}$ and $\beta$ satisfies the following condition

$$
\alpha+M(\alpha-1)<\beta\left(1-\frac{\sqrt{2}}{2} \delta(z)\right) .
$$

Then we obtain

$$
\begin{aligned}
\mid 1-\langle z, \zeta\rangle-\sqrt{(z \bullet z) \overline{(\zeta \bullet \zeta)} \mid} & <\alpha \sqrt{2} \delta(z)+\sqrt{2} M(\alpha-1) \delta(z) \\
& =\sqrt{2} \delta(z)(\alpha+M(\alpha-1)) \\
& <\frac{\beta}{2}\left(1-N_{*}^{2}(z)\right),
\end{aligned}
$$

where the last inequality follows from (2.4) and (2.6). 
We have shown that if $z \in \mathcal{A}_{\alpha}(\zeta)$ and $z$ is close to $\zeta$, then $z \in D_{\beta}^{*}(\zeta)$. This, combined with the identity $\bigcup_{\beta>1} D_{\beta}^{*}(\zeta)=\mathbb{B}_{*}$, proves that there exists $\beta$ large enough which verifies $\mathcal{A}_{\alpha}(\zeta) \subset D_{\beta}^{*}(\zeta), \forall \zeta \in K$.

To prove the second assertion in $(2.2)$ let $\zeta \in K$ and $z \in D_{\alpha}^{*}(\zeta), z$ is close to $\zeta$. Then

$$
\begin{aligned}
|z-\zeta|^{2} \leq|z-\zeta|^{2}+ & |\sqrt{z \bullet z}-\sqrt{\zeta \bullet \zeta}|^{2} \\
& =1+N_{*}^{2}(z)-2 \operatorname{Re}(\langle z, \zeta\rangle+\sqrt{(z \bullet z) \overline{(\zeta \bullet \zeta)}}) .
\end{aligned}
$$

Since $z \in D_{\alpha}^{*}(\zeta)$, we get $\operatorname{Re}(\langle z, \zeta\rangle+\sqrt{(z \bullet z) \overline{(\zeta \bullet \zeta)}})>1-\frac{\alpha}{2}(1-$ $\left.N_{*}^{2}(z)\right)$. Thus

$$
|z-\zeta|^{2} \leq 1+N_{*}^{2}(z)-2\left[1-\frac{\alpha}{2}\left(1-N_{*}^{2}(z)\right)\right]=(\alpha-1)\left(1-N_{*}^{2}(z)\right) .
$$

On account of (2.4) and the last inequality, it follows that $|z-\zeta|^{2}<$ $2 \sqrt{2}(\alpha-1) \delta(z)$. Hence we can choose $\gamma$ such that $\gamma-1>2 \sqrt{2}(\alpha-1)$. It now remains to show that $\left|\left\langle z-\zeta, \nu_{\zeta}\right\rangle\right|\langle\gamma \delta(z)$. The rest of our proof is similar to the previous proof of the first assertion in (2.2), this completes the proposition.

Remark 2.2. Assertion (i) can not be sharpened. None of the two assertions in (2.2) holds if $K$ is replaced by the whole regular part of $\partial \mathbb{B}_{*}$. In other words, the two systems of approach regions are not globally equivalent. This result can be shown by slightly modifying the arguments in the proof of assertion (i).

\section{Analysis on the complex manifold $\mathbb{M}$}

The complex manifold $\mathbb{M}$ is defined by

$$
\mathbb{M}=\mathbb{M}_{n}:=\left\{z \in \mathbb{C}^{n+1} \backslash\{0\}: z \bullet z=0 \text { and }|z|<1\right\} .
$$

The manifold $\partial \mathbb{M}:=\left\{z \in \mathbb{C}^{n+1}: z \bullet z=0\right.$ and $\left.|z|=1\right\}$ is endowed with the unique probability $O(n+1, \mathbb{R})$-invariant measure $\sigma$. This measure is induced by Haar measure of $O(n+1, \mathbb{R})$ (see $[\mathbf{8}])$. Set $\overline{\mathbb{M}}:=\mathbb{M} \cup \partial \mathbb{M}$.

From the work in [19], the Szegö kernel of $\mathbb{M}$ is given by

$$
S_{\mathbb{M}}(z, w)=\frac{1+\langle z, w\rangle}{(1-\langle z, w\rangle)^{n}}, \quad \text { for } z \in \mathbb{M} \text { and } w \in \partial \mathbb{M}
$$


By exploiting this explicit formula, we shall establish the theorems of Fatou, Korányi and Korányi-Vági type on $\mathbb{M}$ using the standard techniques for the unit ball in Rudin's book [16]. The work of Stein $[\mathbf{1 7}$ would not give these results directly since an analogue of the potential theory on Euclidean spaces has not been available yet in the context of the manifold $\mathbb{M}$.

Definition 3.1. For $a \in \overline{\mathbb{M}}, b \in \overline{\mathbb{M}}$,

$$
d(a, b):=|1-\langle a, b\rangle|^{\frac{1}{2}} .
$$

For $w \in \partial \mathbb{M}, \delta>0$,

$$
Q(w, \delta):=\{\eta \in \partial \mathbb{M}: d(w, \eta)<\delta\} .
$$

It is clear that for every $U \in O(n+1, \mathbb{R})$,

$$
d(U a, U b):=d(a, b), \quad \text { and } \quad U(Q(w, \delta))=Q(U w, \delta) .
$$

Observe that $\partial \mathbb{M}$ is a submanifold of the unit sphere of $\mathbb{C}^{n+1}$. Then by Proposition 5.1 .2 of $[\mathbf{1 6}], d$ is a metric on $\partial \mathbb{M}$. Now we define a system of approach regions for $\mathbb{M}$.

Definition 3.2. For $\alpha>1$ and $w \in \partial \mathbb{M}$, we let the approach region $D_{\alpha}(w)$ be the set of all $z \in \mathbb{M}$ such that

$$
|1-\langle z, w\rangle|<\frac{\alpha}{2}\left(1-|z|^{2}\right) \text {. }
$$

The following proposition will be very useful.

Proposition 3.3. There exist two constants $0<C_{1}, C_{2}<\infty$ such that

$$
\forall a \in \partial \mathbb{M} \text { and } 0<\delta<\sqrt{2}: C_{1}<\frac{\sigma(Q(a, \delta))}{\delta^{2 n}}<C_{2} .
$$

Proof: Since $O(n+1, \mathbb{R})$ acts transitively on $\partial \mathbb{M}$ and $\sigma, d, Q(., \delta)$ are $O(n+1, \mathbb{R})$-invariant, we may suppose without loss of generality that $a:=\left(\frac{1}{\sqrt{2}}, \frac{i}{\sqrt{2}}, 0, \ldots, 0\right) \in \partial \mathbb{M}$ and $\delta$ is sufficiently small.

Consider the function $H=\left(H_{1}, \ldots, H_{n}\right): \partial \mathbb{M} \longrightarrow \mathbb{S}^{2 n-1}$ defined by

$$
H_{1}(z):=\frac{\frac{z_{1}}{\sqrt{2}}-\frac{i z_{2}}{\sqrt{2}}}{h(z)}, \quad H_{2}(z):=\frac{z_{3}}{h(z)}, \ldots, H_{n}(z):=\frac{z_{n+1}}{h(z)},
$$

where $\mathbb{S}^{2 n-1}$ is the unit sphere of $\mathbb{C}^{n}$ and

$$
h(z):=\sqrt{\left|\frac{z_{1}}{\sqrt{2}}-\frac{i z_{2}}{\sqrt{2}}\right|^{2}+\left|z_{3}\right|^{2}+\cdots+\left|z_{n+1}\right|^{2}}, \quad z \in \partial \mathbb{M} .
$$

We now prove that $H$ is locally diffeomorphic near the point $a$. 
Write $w=H(z)$. Since $z \in \partial \mathbb{M}$, we have

$$
2 H_{1}(z) \cdot\left(\frac{\frac{z_{1}}{\sqrt{2}}+\frac{i z_{2}}{\sqrt{2}}}{h(z)}\right)+H_{2}^{2}(z)+\cdots+H_{n}^{2}(z)=\frac{z_{1}^{2}+\cdots+z_{n+1}^{2}}{h^{2}(z)}=0 .
$$

Thus

$$
\frac{\frac{z_{1}}{\sqrt{2}}+\frac{i z_{2}}{\sqrt{2}}}{h(z)}=\frac{-w_{2}^{2}-\cdots-w_{n}^{2}}{2 w_{1}} .
$$

This, combined with equation (3.2), gives the following system

$$
\left\{\begin{array}{l}
\left|z_{1}\right|^{2}+\cdots+\left|z_{n+1}\right|^{2}=1 \\
\frac{z_{1}}{h(z)}=\frac{2 w_{1}^{2}-w_{2}^{2}-\cdots-w_{n}^{2}}{2 \sqrt{2} w_{1}} \\
\frac{z_{2}}{h(z)}=\frac{i\left(2 w_{1}^{2}+w_{2}^{2}+\cdots+w_{n}^{2}\right)}{2 \sqrt{2} w_{1}} \\
\frac{z_{3}}{h(z)}=w_{2}, \ldots, \frac{z_{n+1}}{h(z)}=w_{n} .
\end{array}\right.
$$

It follows easily that the equation $w=H(z)$ has a unique solution for every $w$ near $(1,0, \ldots, 0)$. Therefore $H$ is locally diffeomorphic near $a$.

Now, let $Q_{\delta}$ denote the standard nonisotropic ball of radius $\delta$ in $\mathbb{S}^{2 n-1}$ centered at $(1,0, \ldots, 0)$ (See $[\mathbf{1 6}$, p. 65].) We shall prove the following fact

$$
H(Q(a, \delta)) \subset Q_{\sqrt{3} \delta} \quad \text { and } \quad Q_{\delta} \subset H(Q(a, \sqrt{3} \delta)),
$$

provided $\delta$ is sufficiently small.

If $z \in Q(a, \delta)$, then by Definition 3.1 we get $\left|1-\frac{z_{1}}{\sqrt{2}}+\frac{i z_{2}}{\sqrt{2}}\right|<\delta^{2}$. This implies

(3.4) $\left|1-w_{1}\right| \leq\left|1-\frac{z_{1}}{\sqrt{2}}+\frac{i z_{2}}{\sqrt{2}}\right|$

$$
+\left(\frac{1}{h(z)}-1\right) \cdot\left|\frac{z_{1}}{\sqrt{2}}-\frac{i z_{2}}{\sqrt{2}}\right|<\delta^{2}+\left(\frac{1}{h(z)}-1\right) .
$$

On the other hand

$$
\begin{aligned}
& 0 \leq \frac{1}{h(z)}-1=\frac{1-h(z)^{2}}{h(z)(h(z)+1)} \\
& \quad \leq \frac{\left|1-\frac{z_{1}}{\sqrt{2}}+\frac{i z_{2}}{\sqrt{2}}\right|\left(1+\left|\frac{z_{1}}{\sqrt{2}}-\frac{i z_{2}}{\sqrt{2}}\right|\right)}{h(z)(h(z)+1)}<2 \delta^{2},
\end{aligned}
$$

for $z \approx a$, because of $h(a)=1$.

By virtue of (3.4) and (3.5), we conclude that $\left|1-w_{1}\right|<3 \delta^{2}$, which proves the first assertion in (3.3). 
To prove the second assertion in (3.3) let $w \in Q_{\delta}$. Since $H$ is locally diffeomorphic near $a$, we can write $w=H(z)$. Then $\left|H_{1}(z)\right|>1-\delta^{2}$. By virtue of the definition of $H_{1}$ in (3.2) the last inequality implies that

$$
\left|\frac{z_{1}}{\sqrt{2}}-\frac{i z_{2}}{\sqrt{2}}\right|^{2}>\frac{\left(1-\delta^{2}\right)^{2}}{1-\left(1-\delta^{2}\right)^{2}} \cdot\left(\left|z_{3}\right|^{2}+\cdots+\left|z_{n+1}\right|^{2}\right) .
$$

Using this estimate, we obtain

$$
\begin{aligned}
1=\left|z_{1}\right|^{2}+\left|z_{2}\right|^{2}+\cdots+\left|z_{n+1}\right|^{2} & \geq\left|\frac{z_{1}}{\sqrt{2}}-\frac{i z_{2}}{\sqrt{2}}\right|^{2}+\left|z_{3}\right|^{2}+\cdots+\left|z_{n+1}\right|^{2} \\
& >\frac{\left|z_{3}\right|^{2}+\cdots+\left|z_{n+1}\right|^{2}}{1-\left(1-\delta^{2}\right)^{2}} .
\end{aligned}
$$

Thus

(3.6) $\left|z_{3}\right|^{2}+\cdots+\left|z_{n+1}\right|^{2}<1-\left(1-\delta^{2}\right)^{2}$ and $\left|z_{1}\right|^{2}+\left|z_{2}\right|^{2}>\left(1-\delta^{2}\right)^{2}$.

On the one hand, using the second estimate in (3.6) we get

(3.7) $\left|\frac{z_{1}}{\sqrt{2}}-\frac{i z_{2}}{\sqrt{2}}\right|^{2}=\left|z_{1}\right|^{2}+\left|z_{2}\right|^{2}-\left|\frac{z_{1}}{\sqrt{2}}+\frac{i z_{2}}{\sqrt{2}}\right|^{2}>\left(1-\delta^{2}\right)^{2}-\frac{\left|z_{1}+i z_{2}\right|^{2}}{2}$.

On the other hand, using the first estimate in (3.6) we have for $z \approx a$,

$$
\begin{aligned}
\frac{\left|z_{1}+i z_{2}\right|^{2}}{2} & =\frac{\left|z_{1}^{2}+z_{2}^{2}\right|^{2}}{2\left|z_{1}-i z_{2}\right|^{2}}=\frac{\left|z_{3}^{2}+\cdots+z_{n+1}^{2}\right|^{2}}{2\left|z_{1}-i z_{2}\right|^{2}} \\
& \leq \frac{\left(\left|z_{3}\right|^{2}+\cdots+\left|z_{n+1}\right|^{2}\right)^{2}}{2\left|z_{1}-i z_{2}\right|^{2}} \leq \frac{\left(1-\left(1-\delta^{2}\right)^{2}\right)^{2}}{2} \leq 2 \delta^{4} .
\end{aligned}
$$

Putting this estimate into (3.7), we obtain

$$
\left|\frac{z_{1}}{\sqrt{2}}-\frac{i z_{2}}{\sqrt{2}}\right|^{2}>\left(1-\delta^{2}\right)^{2}-2 \delta^{4}>1-3 \delta^{2} .
$$

This implies

$$
0 \leq \frac{1}{h(z)}-1=\frac{1-h(z)^{2}}{h(z)(h(z)+1)} \leq \frac{1-\left|\frac{z_{1}}{\sqrt{2}}-\frac{i z_{2}}{\sqrt{2}}\right|^{2}}{h(z)(h(z)+1)}<2 \delta^{2},
$$

for $z \approx a$. 
Finally, using the last inequality and the assumption that $w \in Q_{\delta}$ we obtain

$\left|1-\frac{z_{1}}{\sqrt{2}}+\frac{i z_{2}}{\sqrt{2}}\right| \leq\left|1-w_{1}\right|+\left(\frac{1}{h(z)}-1\right) \cdot\left|\frac{z_{1}}{\sqrt{2}}-\frac{i z_{2}}{\sqrt{2}}\right|<\delta^{2}+2 \delta^{2}=3 \delta^{2}$.

The proof of (3.3) is now complete.

Next, let $\tau$ denote the Lebesgue surface measure on $\mathbb{S}^{2 n-1}$. Then it is clear that there exists a smooth function $g$ defined in a small neighborhood $U(a)$ of $a$ such that $g(z) \neq 0$ and $d \sigma(z)=g(z) \cdot H^{*} d \tau(z)$ for $z \in U(a)$. We therefore obtain the estimate

$$
\sigma(Q(a, \delta))=\int_{Q(a, \delta)} g \cdot H^{*} d \tau \leq \max _{z \in U(a)}|g| \cdot \int_{Q_{\sqrt{3} \delta}} d \tau<C_{2} \delta^{2 n},
$$

where the first inequality follows from the first assertion in (3.3) and the second one from Proposition 5.1.4 of [16].

A similar argument using the second assertion in (3.3) shows that $\sigma(Q(a, \delta))>C_{1} \delta^{2 n}$.

The proof of Proposition 3.3 is complete.

Remark 3.4. An immediate consequence of the above proposition is that the triple $(\partial \mathbb{M}, \sigma, d)$ is a space of homogeneous type.

Proposition 3.5. If $\eta \in \partial \mathbb{M}, \zeta \in \partial \mathbb{M}, z \in D_{\alpha}(\zeta),|z|=r$, then

$$
|1-\langle\eta, \zeta\rangle|<4 \alpha|1-\langle z, \eta\rangle|
$$

and

$$
P_{\mathbb{M}}(z, \eta)<(4 \alpha)^{2 n} \frac{(1+r)^{2}\left(1-r^{2}\right)^{n}}{1+r^{2}} \cdot \frac{1}{[d(\zeta, \eta)]^{4 n}} .
$$

Proof: Note that $\partial \mathbb{M} \subset \mathbb{S}^{2 n+1}$. Then the first estimate follows from Lemma 5.3 .4 of $[\mathbf{1 6}]$.

From (3.1), the Poisson-Szegö kernel of $\mathbb{M}$ is given by

(3.8) $P_{\mathbb{M}}(z, w)=\frac{|1+\langle z, w\rangle|^{2}}{|1-\langle z, w\rangle|^{2 n}} \cdot \frac{(1-\langle z, z\rangle)^{n}}{(1+\langle z, z\rangle)}, \quad$ for $z \in \mathbb{M}$ and $w \in \partial \mathbb{M}$.

Applying the first estimate to the above formula, our second estimate follows.

Definition 3.6. If $u \in \mathcal{C}(\mathbb{M})$ and $\alpha>1$, the maximal function $M_{\alpha} u$ : $\partial \mathbb{M} \rightarrow[0, \infty]$ is defined by

$$
\left(M_{\alpha} u\right)(w):=\sup \left\{|u(z)|: z \in D_{\alpha}(w)\right\} .
$$


If $\mu$ is a complex measure on $\partial \mathbb{M}, M \mu$ denotes its radial HardyLittlewood maximal function in the space $(\partial \mathbb{M}, \sigma, d), \mathcal{S}_{\mathbb{M}}[\mu]$ is its Szegö integral and $\mathcal{P}_{\mathbb{M}}[\mu]$ is its Poisson-Szegö integral.

The following theorem can be regarded as Korányi type theorem on $\mathbb{M}$.

Theorem 3.7. To every $\alpha>1$ corresponds a constant $C(\alpha)<\infty$ such that the inequality

$$
M_{\alpha} \mathcal{P}_{\mathbb{M}}[\mu] \leq C(\alpha) M \mu
$$

holds for every complex measure $\mu$ on $\partial \mathbb{M}$.

Proof: We apply Propositions 3.3 and 3.5. The proof given in Theorem 5.4.5 of [16] is valid in the context of the space of homogeneous type $(\partial \mathbb{M}, \sigma, d)$ making the obviously necessary changes.

The arguments which have been used in Theorems 5.4.9 and 5.4.10 of [16] give the following

Proposition 3.8. If $\mu$ is a complex measure on $\partial \mathbb{M}$ and $\mathcal{D} \mu$ denotes its derivative in the space $(\partial \mathbb{M}, \sigma, d)$, then $\mathcal{P}_{\mathbb{M}}[\mu]$ has finite admissible limits $\left(\mathcal{P}_{\mathbb{M}}[\mu]\right)^{*}$ a.e. $[\sigma]$ on $\partial \mathbb{M}$ and

$$
\left(\mathcal{P}_{\mathbb{M}}[\mu]\right)^{*}(w)=(\mathcal{D} \mu)(w) \quad \text { a.e. }[\sigma] \text { on } \partial \mathbb{M} \text {. }
$$

If moreover $1<p<\infty$ and $f \in L^{p}(\partial \mathbb{M}, \sigma)$, then

$$
\int_{\partial \mathbb{M}}\left|M_{\alpha} \mathcal{P}_{\mathbb{M}}[f]\right|^{p} d \sigma \leq C(\alpha, p) \int_{\partial \mathbb{M}}|f|^{p} d \sigma .
$$

Definition 3.9. For $\zeta, \omega \in \partial \mathbb{M}, \alpha>1, \delta>0$, we define a maximal difference

$$
\Delta(\zeta, \omega, \alpha, \delta):=\sup \left|S_{\mathbb{M}}(z, \eta)-S_{\mathbb{M}}(z, \omega)\right|,
$$

the supremum being taken over all $\eta \in Q(\omega, \delta)$ and over all $z \in D_{\alpha}(\zeta)$.

Theorem 3.10. If $\omega \in \partial \mathbb{M}, \alpha>1, \delta>0$, then

$$
\int_{R(\omega, \delta)} \Delta(\zeta, \omega, \alpha, \delta) d \sigma(\zeta)<C(\alpha),
$$

where $R(\omega, \delta):=\partial \mathbb{M} \backslash Q(\omega, 2 \delta)$.

Proof: First observe that in fact the method given in Lemma 6.1.1 of [16] proves the following 
Fact. Suppose $\zeta, \eta, \omega \in \partial \mathbb{M} \subset \mathbb{S}^{2 n+1}, d(\omega, \eta)<\delta, d(\omega, \zeta)>2 \delta$, and $z \in D_{\alpha}(\zeta)$. Then for every $k \in \mathbb{N}$ we have

$$
\left|\frac{1}{(1-\langle z, \eta\rangle)^{k}}-\frac{1}{(1-\langle z, \omega\rangle)^{k}}\right| \leq(16 \alpha)^{k+1} \delta|1-\langle\zeta, \omega\rangle|^{-k-\frac{1}{2}} .
$$

Combining this fact, formula (3.1) and Definition 3.9, we get

$$
\begin{aligned}
\Delta(\zeta, \omega, \alpha, \delta) \leq & 2 \sup \left|\frac{1}{(1-\langle z, \eta\rangle)^{n}}-\frac{1}{(1-\langle z, \omega\rangle)^{n}}\right| \\
& +\sup \left|\frac{1}{(1-\langle z, \eta\rangle)^{n-1}}-\frac{1}{(1-\langle z, \omega\rangle)^{n-1}}\right| \\
\leq & 2(16 \alpha)^{n+1} \delta|1-\langle\zeta, \omega\rangle|^{-n-\frac{1}{2}}+(16 \alpha)^{n} \delta|1-\langle\zeta, \omega\rangle|^{-n+\frac{1}{2}} .
\end{aligned}
$$

Therefore,

$$
\Delta(\zeta, \omega, \alpha, \delta) \leq 2(16 \alpha)^{n}(1+16 \alpha) \delta|1-\langle\zeta, \omega\rangle|^{-n-\frac{1}{2}}
$$

On account of (3.12), estimate (3.11) is reduced to proving the following inequality

$$
\int_{R(\omega, \delta)}|1-\langle\zeta, \omega\rangle|^{-n-\frac{1}{2}} d \sigma(\zeta)<\frac{C}{\delta} .
$$

We now apply the arguments of Proposition 3.3: Suppose without loss of generality that $\omega:=\left(\frac{1}{\sqrt{2}}, \frac{i}{\sqrt{2}}, 0, \ldots, 0\right) \in \partial \mathbb{M}$ and $\delta<\delta_{0}, \delta_{0}$ is sufficiently small. By virtue of (3.3), we get

$$
\begin{aligned}
& \int_{Q\left(\omega, 2 \delta_{0}\right) \backslash Q(\omega, 2 \delta)}|1-\langle\zeta, \omega\rangle|^{-n-\frac{1}{2}} d \sigma(\zeta) \\
\leq & \int_{Q\left(\omega, 2 \delta_{0}\right) \backslash H^{-1}}\left(Q_{\frac{2}{\sqrt{3}} \delta}\right) \\
\leq & 3^{n+\frac{1}{2}}|1-\langle H(\zeta), H(\omega)\rangle|^{-n-\frac{1}{2}} d \sigma(\zeta) \\
& \int_{\mathbb{S}^{2 n-1} \backslash Q_{\frac{2}{\sqrt{3}} \delta}}\left|1-\eta_{1}\right|^{-n-\frac{1}{2}} d \tau(\eta) \leq \frac{C}{\delta},
\end{aligned}
$$

where in the last inequality we apply Theorem 6.1 .3 of [16].

Thus inequality (3.13) is proved, and the theorem is thereby concluded. 
Proposition 3.11. If $1 \leq p \leq \infty, f \in L^{p}(\partial \mathbb{M}, \sigma), u=\mathcal{P}_{\mathbb{M}}[f]$, and

$$
u_{r}(w):=u(r w) \quad(0 \leq r<1, w \in \partial \mathbb{M})
$$

then $\left\|u_{r}\right\|_{L^{p}(\partial \mathbb{M}, \sigma)} \leq\|f\|_{L^{p}(\partial \mathbb{M}, \sigma)}$.

If also $p<\infty$ then

$$
\lim _{r \rightarrow 1^{-}}\left\|u_{r}-f\right\|_{L^{p}(\partial \mathbb{M}, \sigma)}=0 .
$$

Proof: The proofs of Proposition 3.3.3 and Theorem 3.3.4 in [16] work with trivial changes making use of formula (3.8).

Theorem 3.12. Suppose $F \in H^{p}(\mathbb{M}, \sigma)$.

(i) If $p>1$ then there is a $f \in L^{p}(\partial \mathbb{M}, \sigma)$ such that $F=\mathcal{P}_{\mathbb{M}}[f]$.

(ii) If $p=1$ then there is a measure $\mu$ on $\partial \mathbb{M}$ such that $F=\mathcal{P}_{\mathbb{M}}[\mu]$.

Proof: We shall indicate briefly that the proof of Theorem 4.3 .3 in [16] works. The fact that $S O(n+1, \mathbb{R})$ acts transitively on $\partial \mathbb{M}$ plays a crucial role here. Instead of the unitary group we now use the group $S O(n+1, \mathbb{R})$ endowed with its Haar measure $d \mathcal{U}$. We define

$$
G(z):=\int_{S O(n+1, \mathbb{R})} F(U z) h(U) d \mathcal{U}, \quad \forall z \in \mathbb{M},
$$

where $h: S O(n+1, \mathbb{R}) \rightarrow[0, \infty)$ is a continuous function that satisfies $\int_{S O(n+1, \mathbb{R})} h(U) d \mathcal{U}=1$.

Similarly as in [16, p. 57], we establish that there is a sequence $r_{i} \rightarrow 1$ such that the dilated functions $\left\{G_{r_{i}}\right\}$ of $G$ converges uniformly to a function $g \in \mathcal{C}(\partial \mathbb{M})$ i.e. $\epsilon_{i}=\sup \left|G\left(r_{i} w\right)-g(w)\right| \rightarrow 0$.

Since $G_{r_{i}} \in A(\mathbb{M})(:=\mathcal{C}(\overline{\mathbb{M}}) \cap H(\mathbb{M}))$, we obtain, by the reproducing property of the Poisson-Szegö kernel,

$$
G\left(r_{i} z\right)=\int_{\partial \mathbb{M}} P_{\mathbb{M}}(z, w) G\left(r_{i} w\right) d \sigma(w), \quad \forall z \in \mathbb{M} .
$$

Thus

$$
\begin{aligned}
\left|G\left(r_{i} z\right)-\mathcal{P}_{\mathbb{M}}[g](z)\right| & =\left|\int_{\partial \mathbb{M}} P_{\mathbb{M}}(z, w)\left(G\left(r_{i} w\right)-g(w)\right) d \sigma(w)\right| \\
& \leq \epsilon_{i} \cdot \int_{\partial \mathbb{M}} P_{\mathbb{M}}(z, w) d \sigma(w)=\epsilon_{i} \rightarrow 0 .
\end{aligned}
$$

This implies that $G(z)=\mathcal{P}_{\mathbb{M}}[g](z)$ for all $z \in \mathbb{M}$. 
The rest of the proof follows the same lines as that given in $[\mathbf{1 6}$, pp. 57-58].

The following result can be regarded as Fatou type theorem on $\mathbb{M}$.

Theorem 3.13. (i) To every $p>1, \alpha>1$ corresponds a constant $C(\alpha, p)<\infty$ such that

$$
\int_{\partial \mathbb{M}}\left|M_{\alpha} f\right|^{p} d \sigma \leq C(\alpha, p)\|f\|_{H^{p}(\mathbb{M}, \sigma)}^{p}
$$

for every $f \in H^{p}(\mathbb{M}, \sigma)$.

(ii) Suppose that $1 \leq p<\infty, f \in H^{p}(\mathbb{M}, \sigma)$ and let $f^{*}$ denote the admissible limits of $f$. Then $f^{*}(w)$ exists a.e. on $\partial \mathbb{M}$ and moreover when $p>1$ we have

$$
\lim _{r \rightarrow 1^{-}} \int_{\partial \mathbb{M}}\left|f^{*}(w)-f(r w)\right|^{p} d \sigma(w)=0 .
$$

Proof: Consider $f \in H^{p}(\mathbb{M}, \sigma)(p \geq 1)$. According to Theorem 3.12, there exists a function $\mu \in L^{p}(\partial \mathbb{M}, \sigma)$ (or a complex measure if $p=1$ ) such that $f=\mathcal{P}_{\mathbb{M}}[\mu]$. By virtue of Proposition 3.11, $\|f\|_{H^{p}(\mathbb{M}, \sigma)}=$ $\|\mu\|_{L^{p}(\partial \mathbb{M}, \sigma)}$. Therefore part (i) follows from estimate (3.10) in Proposition 3.8.

Applying equation (3.9) of Proposition 3.8 to the equation $f=\mathcal{P}_{\mathbb{M}}[\mu]$, we obtain $f^{*}=\mathcal{D} \mu$. The proof of Theorem 5.6.6 in [16] also works using part (i). This finishes part (ii).

Theorem 3.14. (i) If $p>1$ and $f \in H^{p}(\mathbb{M}, \sigma)$ then $f^{*} \in H^{p}(\partial \mathbb{M}, \sigma)$ and $f=\mathcal{S}_{\mathbb{M}}\left[f^{*}\right]=\mathcal{P}_{\mathbb{M}}\left[f^{*}\right]$. Here $H^{p}(\partial \mathbb{M}, \sigma)$ denotes the closure of the algebra $A(\mathbb{M})$ in $L^{p}(\partial \mathbb{M}, \sigma)$.

(ii) The map $f \longrightarrow\left(\mathcal{S}_{\mathbb{M}}[f]\right)^{*}$ is the orthogonal projection of $L^{2}(\partial \mathbb{M}, \sigma)$ onto $H^{2}(\partial \mathbb{M}, \sigma)$, and

$$
\int_{\partial \mathbb{M}}\left|M_{\alpha} \mathcal{S}_{\mathbb{M}}[f]\right|^{2} d \sigma \leq C(\alpha) \int_{\partial \mathbb{M}}|f|^{2} d \sigma
$$

for every $f \in L^{2}(\partial \mathbb{M}, \sigma)$.

Proof: The proofs of Theorems 5.6.8 and 5.6.9 in [16] are also valid in this context making use of Theorem 3.13.

Finally, combining Theorem 3.10 and part (ii) of Theorem 3.14, the arguments which have been used in Theorems 6.2 .2 and 6.3 .1 of [16] prove the following Korányi-Vági type theorem on $\mathbb{M}$. 
Theorem 3.15. If $1<p<\infty$ and $\alpha>1$, there exists a constant $C(\alpha, p)<\infty$ such that

$$
\int_{\partial \mathbb{M}}\left|M_{\alpha} \mathcal{S}_{\mathbb{M}}[f]\right|^{p} d \sigma \leq C(\alpha, p) \int_{\partial \mathbb{M}}|f|^{p} d \sigma
$$

for every $f \in L^{p}(\partial \mathbb{M}, \sigma)$.

If $p=1$ and $\alpha>1$, there exists a constant $C(\alpha, 1)<\infty$ such that for every $\lambda>0$ and for every complex measure $\mu$ on $\partial \mathbb{M}$,

$$
\sigma\left\{w \in \partial \mathbb{M}:\left|\left(M_{\alpha} \mathcal{S}_{\mathbb{M}}[\mu]\right)(w)\right|>\lambda\right\} \leq C(\alpha, 1) \frac{\|\mu\|}{\lambda} .
$$

\section{Proofs of the main theorems}

In what follows we shall use the function $F$ as well as its two local inverses $\phi$ and $\psi$ introduced in [12, p. 919]. In this section we transplant the results established in Section 3 to the minimal ball by means of the proper holomorphic mapping $F$.

If $f: \mathbb{B}_{*} \backslash\{0\} \longrightarrow \mathbb{C}$ is a measurable function and if $z=\left(z_{1}, \ldots, z_{n+1}\right) \in$ $\mathbb{M}$, we set

$$
(T f)(z):=z_{n+1}(f \circ F)(z)=z_{n+1} f\left(z_{1}, \ldots, z_{n}\right) .
$$

We recall the following identities from the work in [19]:

$$
\phi^{*}(d \sigma)(\zeta)=\psi^{*}(d \sigma)(\zeta)=\frac{C_{3}}{2} \cdot \frac{d \theta(\zeta)}{|\zeta \bullet \zeta|}, \quad \text { for } \zeta \in \partial \mathbb{B}_{*} \backslash V,
$$

where $C_{3}$ is a constant that depends only on the dimension $n$, and

$$
\mathcal{S}_{\mathbb{M} \circ} \circ T[f]=T \circ \mathcal{S}_{\mathbb{B}_{*}}[f], \quad \text { for } f \in L^{2}\left(\partial \mathbb{B}_{*}, \theta\right) .
$$

In view of Definitions 1.1 and 3.2, we deduce that

$$
D_{\alpha}^{*}(\zeta)=F\left(D_{\alpha}(\phi(\zeta))\right)=F\left(D_{\alpha}(\psi(\zeta))\right), \quad \forall \zeta \in \partial \mathbb{B}_{*} \backslash V .
$$

We now complete the proof of Proposition 2.1.

Proof of assertion (ii) of Proposition 2.1: Since the approach regions on $\mathbb{M}$ are the Korányi approach regions on the unit ball of $\mathbb{C}^{n+1}$ restricted on $\mathbb{M}, D_{\alpha}(\zeta)$ is tangential to $\partial \mathbb{M}$ in the direction of $T_{\zeta}^{\mathbb{C}}(\partial \mathbb{M})$ for $\alpha>1$ and $\zeta \in \partial \mathbb{M}$. In addition, $F$ is locally biholomorphic. Therefore assertion (ii) of Proposition 2.1 follows from identity (4.4).

The following lemma will be very useful. 
Lemma 4.1. For each $p>-1$, there exist two constants $0<C_{4}(p)$, $C_{5}(p)<\infty$ such that

$$
C_{4}<\frac{\int_{Q(a, \delta)}\left|w_{n+1}\right|^{2 p} d \sigma(w)}{\delta^{2 n+2 p}}<C_{5},
$$

where $a=\left(a_{1}, \ldots, a_{n+1}\right) \in \partial \mathbb{M}$ with $a_{n+1}=0$ and $0<\delta<\sqrt{2}$.

Proof: Observe that $\partial \mathbb{M}_{n-1}=\left\{a \in \partial \mathbb{M}_{n}: a_{n+1}=0\right\}$. Since $S O(n, \mathbb{R})$ acts transitively on $\partial \mathbb{M}_{n-1}$, we may suppose without loss of generality that $a:=\left(\frac{1}{\sqrt{2}}, \frac{i}{\sqrt{2}}, 0, \ldots, 0\right) \in \partial \mathbb{M}$ as in the proof of Proposition 3.3.

Using the local diffeomorphism $H$ constructed in (3.2) together with its properties (3.3), inequality (4.5) is reduced to proving the following estimate

$$
C_{4}<\frac{\int_{Q_{\delta}}\left|\zeta_{n}\right|^{2 p} d \tau(\zeta)}{\delta^{2 n+2 p}}<C_{5} .
$$

We now prove (4.6). Applying formula 1.4.4(1) in [16], we get

$$
\begin{aligned}
& \int_{Q_{\delta}}\left|\zeta_{n}\right|^{2 p} d \tau(\zeta) \\
& \quad=\left(\begin{array}{c}
n-1 \\
2
\end{array}\right) \int_{B_{2}}\left(1-\left|\left(\lambda_{1}, \lambda_{2}\right)\right|^{2}\right)^{n-3} \chi_{\left\{\left|1-\lambda_{1}\right|<\delta^{2}\right\}}\left|\lambda_{2}\right|^{2 p} d \nu_{2}\left(\lambda_{1}, \lambda_{2}\right),
\end{aligned}
$$

where $\chi$ is the characteristic function, $B_{2}$ is the unit ball of $\mathbb{C}^{2}$ and $\nu_{2}$ is the Lebesgue measure on $\mathbb{C}^{2}$ so normalized that $\nu_{2}\left(B_{2}\right)=1$.

Fubini's theorem shows that the right side of the last equation equals

$$
\begin{aligned}
& C \cdot \int_{\mathbb{C}} \chi_{\left\{\left|\lambda_{1}\right|<1,\left|1-\lambda_{1}\right|<\delta^{2}\right\}} d m\left(\lambda_{1}\right) \\
& \cdot \int_{\left|\lambda_{2}\right| \leq \sqrt{1-\left|\lambda_{1}\right|^{2}}}\left(1-\left|\lambda_{1}\right|^{2}-\left|\lambda_{2}\right|^{2}\right)^{n-3}\left|\lambda_{2}\right|^{2 p} d m\left(\lambda_{2}\right),
\end{aligned}
$$

where $m$ is the ordinary Lebesgue measure of $\mathbb{C}$. 
Using the beta function, since $p>-1$, the inner integral of the expression above is equal to

$$
C\left(1-\left|\lambda_{1}\right|^{2}\right)^{n+p-2} B(n-2, p+1) .
$$

Hence we get

$$
\int_{Q_{\delta}}\left|\zeta_{n}\right|^{2 p} d \tau(\zeta)=C \cdot \int_{E(\delta)}\left(1-\left|\lambda_{1}\right|^{2}\right)^{n+p-2} d m\left(\lambda_{1}\right),
$$

where $E(\delta):=\left\{\lambda_{1} \in \mathbb{C}:\left|\lambda_{1}\right|<1\right.$ and $\left.\left|1-\lambda_{1}\right|<\delta^{2}\right\}$.

In view of (4.7), the arguments which have been used in Proposition 5.1.4 of [16] establish (4.6). Therefore, the proof of the lemma is complete.

Proof of Theorem A: Take any $k \in \mathbb{N}$ such that $k p>2 s+2$. Consider the function $g \in H(\mathbb{M})$ defined by

$$
g(z):=z_{n+1}^{k} f(F(z)), \quad \forall z \in \mathbb{M} .
$$

By virtue of formula (4.2) we have, for $0<r<1$,

$$
\begin{aligned}
\int_{\partial \mathbb{M}}|g(r w)|^{p} d \sigma(w) \leq \int_{\partial \mathbb{M}}\left|w_{n+1}\right|^{2 s+2}|f(r F(w))|^{p} d \sigma(w) \\
=C_{3} \cdot \int_{\partial \mathbb{B}_{*}}|f(r \zeta)|^{p}|\zeta \bullet \zeta|^{s} d \theta(\zeta)<\infty,
\end{aligned}
$$

since $f \in H^{p}\left(\mathbb{B}_{*},|\zeta \bullet \zeta|^{s} d \theta\right)$.

The latter estimate gives that $g \in H^{p}(\mathbb{M}, \sigma)$. By part (ii) of Theorem 3.13 the boundary value $g^{*}(w)$ exists almost everywhere with respect to the measure $\sigma$ on $\partial \mathbb{M}$. This, combined with formula (4.2), identity (4.4) and equation (4.8) gives that $f$ has admissible limits almost everywhere with respect to the measure $\theta$ on $\partial \mathbb{B}_{*}$.

Since $r \zeta \in D_{\alpha}^{*}(\zeta)$ for $0 \leq r<1$ and $\alpha>2$, the admissible convergence of $f$ implies that the dilated functions $f_{r}$ converge to $f^{*} \theta$-almost everywhere on $\partial \mathbb{B}_{*}$. Therefore it follows from Fatou's lemma that $f^{*} \in L^{p}\left(\partial \mathbb{B}_{*},|\zeta \bullet \zeta|^{s} d \theta\right)$. This completes the proof of part (i). 
Taking Theorem B for granted, we now prove part (ii). By part (ii) of Theorem B, we have

$$
\lim _{r \rightarrow 1^{-}} \int_{\partial \mathbb{B}_{*}}\left|f(r \zeta)-f^{*}(\zeta)\right|^{p}|\zeta \bullet \zeta|^{s} d \theta(\zeta)=0
$$

Using (4.9) and applying the Hölder's inequality, we see that for each $z \in \mathbb{B}_{*}$, there exists a constant $C(z)<\infty$ such that

$$
\begin{aligned}
\mid \mathcal{S}_{\mathbb{B}_{*}}\left[f^{*}\right](z)- & f(z)\left|=\lim _{r \rightarrow 1^{-}}\right| \mathcal{S}_{\mathbb{B}_{*}}\left[f^{*}-f_{r}\right](z) \mid \\
\leq & \left(\int_{\partial \mathbb{B}_{*}}\left|S_{\mathbb{B}_{*}}(z, \zeta)\right|^{\frac{p}{p-1}}|\zeta \bullet \zeta|^{\frac{-s}{p-1}} d \theta(\zeta)\right)^{\frac{p-1}{p}} \\
& \cdot \lim _{r \rightarrow 1^{-}}\left(\int_{\partial \mathbb{B}_{*}}\left|f(r \zeta)-f^{*}(\zeta)\right|^{p}|\zeta \bullet \zeta|^{s} d \theta(\zeta)\right)^{\frac{1}{p}} \\
\leq & C(z)\left(\int_{\partial \mathbb{M}^{2}}\left|w_{n+1}\right|^{2-\frac{2 s}{p-1}} d \sigma(w)\right)^{\frac{p-1}{p}} \\
& \cdot \lim _{r \rightarrow 1^{-}}\left(\int_{\partial \mathbb{B}_{*}}\left|f(r \zeta)-f^{*}(\zeta)\right|^{p}|\zeta \bullet \zeta|^{s} d \theta(\zeta)\right)^{\frac{1}{p}} \\
\leq & C \cdot C(z) \lim _{r \rightarrow 1^{-}}\left(\int_{\partial \mathbb{B}_{*}}\left|f(r \zeta)-f^{*}(\zeta)\right|^{p}|\zeta \bullet \zeta|^{s} d \theta(\zeta)\right)^{\frac{1}{p}}=0,
\end{aligned}
$$

where the second inequality follows from formula (4.2) and the third one comes from Lemma 4.1 and the hypothesis on $s$.

This yields $f=\mathcal{S}_{\mathbb{B}_{*}}\left[f^{*}\right]$. The identity $f=\mathcal{P}_{\mathbb{B}_{*}}\left[f^{*}\right]$ can be proved in the same way.

In order to prove Theorems B and C we need some lemmas. 
We recall from the work of Calderón [2] that the weight function $\mu(w)$, $\mu(w) \geq 0$, is said to belong to the class $A_{p}$ of Muckenhoupt $(1<p<\infty)$ if

where

$$
\sup _{a \in \partial \mathbb{M}, 0<\delta<\sqrt{2}} M(\mu, a, \delta)<\infty
$$

$$
\begin{aligned}
M(\mu, a, \delta):= & \left\{\frac{1}{\sigma(Q(a, \delta))} \int_{Q(a, \delta)} \mu(w) d \sigma(w)\right\} \\
& \cdot\left\{\frac{1}{\sigma(Q(a, \delta))} \int_{Q(a, \delta)} \mu(w)^{\frac{-1}{p-1}} d \sigma(w)\right\}^{p-1} .
\end{aligned}
$$

Lemma 4.2. Consider the weight function $\mu_{s}(w):=\left|w_{n+1}\right|^{2 s}, s \in \mathbb{R}$, in the space of homogeneous type $(\partial \mathbb{M}, \sigma, d)$. Then $\mu_{s}$ belongs to the class $A_{p}$ of Muckenhoupt if $-1<s<p-1$.

Proof: Take two sequences $\left\{a^{k}\right\} \subset \partial \mathbb{M}$ and $\left\{\delta_{k}\right\} \subset \mathbb{R}^{+}$such that

- $\sup _{a \in \partial \mathbb{M}, 0<\delta<\sqrt{2}} M\left(\mu_{s}, a, \delta\right)=\lim _{k \rightarrow \infty} M\left(\mu_{s}, a^{k}, \delta_{k}\right)$;

- $\lim _{k \rightarrow \infty} a^{k}=a^{0}, \lim _{k \rightarrow \infty} \delta_{k}=\delta_{0}$.

There are three cases to consider.

Case (1): $\delta_{0}>0$. Then

$$
\sup _{a \in \partial \mathbb{M}, 0<\delta<\sqrt{2}} M\left(\mu_{s}, a, \delta\right)=M\left(\mu_{s}, a^{0}, \delta_{0}\right) .
$$

Since $s>-1$ and $\frac{-s}{p-1}>-1$, it follows from Lemma 4.1 that $\int_{Q\left(a^{0}, \delta_{0}\right)} \mu(w) d \sigma(w)<\infty$ and $\int_{Q\left(a^{0}, \delta_{0}\right)} \mu(w)^{\frac{-1}{p-1}} d \sigma(w)<\infty$. Hence $M\left(\mu_{s}, a^{0}, \delta_{0}\right)<\infty$.

Case (2): $\delta_{0}=0$ and $a^{0} \in \partial \mathbb{M}_{n-1}$. Then

$$
\sup _{a \in \partial \mathbb{M}, 0<\delta<\sqrt{2}} M\left(\mu_{s}, a, \delta\right) \leq \limsup _{\delta \rightarrow 0} M\left(\mu_{s}, a^{0}, \delta\right) .
$$

Applying Lemma 4.1 to the equation (4.11), we obtain

$$
\limsup _{\delta \rightarrow 0} M\left(\mu_{s}, a^{0}, \delta\right) \leq C_{5}(s)\left[C_{5}\left(\frac{-s}{p-1}\right)\right]^{p-1}<\infty .
$$


Case (3): $\delta_{0}=0$ and $a^{0} \notin \partial \mathbb{M}_{n-1}$. It is easy to see that

$$
\lim _{\delta \rightarrow 0} M\left(\mu_{s}, a^{0}, \delta\right)=\left|a_{n+1}^{0}\right|^{2 s}\left(\left|a_{n+1}^{0}\right|^{\frac{-2 s}{p-1}}\right)^{p-1}=1 .
$$

In any case we always have $\sup _{a \in \partial \mathbb{M}, 0<\delta<\sqrt{2}} M\left(\mu_{s}, a, \delta\right)<\infty$, this establishes the lemma.

If $u \in \mathcal{C}(\mathbb{M})$, the radial maximal function $M_{\mathrm{rad}} u: \partial \mathbb{M} \rightarrow[0, \infty]$ is defined by

$$
\left(M_{\mathrm{rad}} u\right)(w):=\sup _{\frac{1}{2}<r<1}|u(r w)|, \quad \forall w \in \partial \mathbb{M}
$$

Lemma 4.3. To every $p>0$ and $\alpha>1$ corresponds a constant $C(\alpha, p)<$ $\infty$ such that

$$
\left|\left(M_{\alpha} u\right)(w)\right|^{p}<C(\alpha, p) \cdot M\left(\left|M_{\mathrm{rad}} u\right|^{p}\right)(w),
$$

for every $u \in H(\mathbb{M})$ and $w \in \partial \mathbb{M}$.

Proof: For $z \in \mathbb{M}$, let $T_{z}$ be the complex tangent space to $\mathbb{M}$ at $z$ and let $\pi_{z}$ be the orthogonal projection of $\mathbb{C}^{n+1}$ onto $T_{z}$. Write $z=r \zeta, \zeta \in \partial \mathbb{M}$. Pick the vectors $\zeta_{2}, \ldots, \zeta_{n}, \zeta_{n+1}$ so that $\left\{\zeta, \zeta_{2}, \ldots, \zeta_{n}\right\}$ is an orthonormal basis for $T_{z}$ and $\left\{\zeta, \zeta_{2}, \ldots, \zeta_{n}, \zeta_{n+1}\right\}$ is an orthonormal basis for $\mathbb{C}^{n+1}$. For any $\delta>0$, consider

$$
\begin{aligned}
& P(z, \delta):=\left\{w=r \zeta+\lambda \zeta+\sum_{j=2}^{n+1} \lambda_{j} \zeta_{j}:|\lambda|<\delta,\left|\lambda_{j}\right|<\delta^{\frac{1}{2}}, j=2, \ldots, n+1\right\}, \\
& \widetilde{P}(z, \delta):=\left\{w=r \zeta+\lambda \zeta+\sum_{j=2}^{n} \lambda_{j} \zeta_{j}:|\lambda|<\delta,\left|\lambda_{j}\right|<\delta^{\frac{1}{2}}, j=2, \ldots, n\right\} \subset T_{z} .
\end{aligned}
$$

The polydiscs $P(z, \delta)$ were considered in the work of Ahern-Bruna [1, p. 132]. Since $\partial \mathbb{M}$ is a subset of the unit sphere of $\mathbb{C}^{n+1}$, it follows from Lemma 3.5 of $[\mathbf{1}]$ that for each $\alpha, \beta, 1<\alpha<\beta$, there is an $\epsilon_{0}>0$ such that if $\eta, \zeta \in \partial \mathbb{M}$ and $z=r \zeta \in D_{\alpha}(\eta)$ then

$$
\begin{aligned}
& P\left(z, \epsilon_{0}(1-\right.\left.\left.r^{2}\right)\right) \\
& \subset \mathbb{M} \\
& \subset\left\{z \in D_{\beta}(\eta): \frac{1}{2}\left(1-r^{2}\right)<1-|z|^{2}<2\left(1-r^{2}\right)\right\} .
\end{aligned}
$$


Fix a point $z_{0} \in \partial \mathbb{M}$. In a sufficiently small compact neighborhood $\mathcal{U}$ of $z_{0}$ in $\overline{\mathbb{M}}$, we can choose the vectors $\zeta_{2}, \ldots, \zeta_{n}, \zeta_{n+1}$ so that they all depend smoothly on $z \in \mathcal{U}$. By shrinking $\mathcal{U}$ we see that there exists an $\delta_{0}>0$ such that

$$
\widetilde{P}(z, \delta) \subset \pi_{z}(P(z, 2 \delta) \cap \mathbb{M}), \quad \forall z \in \mathcal{U}, 0<\delta<\delta_{0} .
$$

By Lemma 2.5 of $[\mathbf{1}]$, we have

$$
\begin{array}{r}
|u(z)|^{p} \leq \frac{C}{\delta^{n+1}} \int_{\widetilde{P}(z, \delta)}\left|\left(u \circ\left(\pi_{z}\right)^{-1}\right)(\zeta)\right|^{p} d V_{z}(\zeta), \\
\text { for } z \in \mathcal{U} \text { and } 0<\delta<\delta_{0} .
\end{array}
$$

Here $d V_{z}$ is the Lebesgue measure on the hyperplane $T_{z}$ and the constant $C$ is independant of $z \in \mathcal{U}$.

Choosing $\epsilon:=\min \left\{\epsilon_{0}, \delta_{0}\right\}$, it follows from (4.14) and (4.15) that

$$
|u(z)|^{p} \leq \frac{C}{(1-r)^{n+1}} \int_{P\left(z, \epsilon\left(1-r^{2}\right)\right) \cap \mathbb{M}}|u(w)|^{p} d V(w), \quad \text { for } z \in \mathcal{U}
$$

Here $d V$ is the surface measure on the complex manifold $\mathbb{M}$ and the constant $C$ is independant of $z \in \mathcal{U}$.

Since the group $O(n+1, \mathbb{R})$ acts transitively on $\partial \mathbb{M}$ and $d V$ is $O(n+$ $1, \mathbb{R})$-invariant, we conclude that (4.16) also holds for all $z \in \mathbb{M}$ sufficiently close to $\partial \mathbb{M}$. On the other hand this estimate is semi-trivial if $z \in \mathbb{M}$ satisfies $|z|<r_{0}$, for some $r_{0}<1$ fixed. Hence (4.16) is valid for all $z \in \mathbb{M}$.

On account of (4.13), (4.16) and by Proposition 3.3, the lemma is proved exactly as in Lemma 4.4 of $[\mathbf{1}]$.

We now come to the the proof of Theorem B.

Proof of Theorem B: We begin the proof of part (i) by choosing $m \in \mathbb{N}$ such that $\frac{m p}{2}-2<s<\left(\frac{m}{2}+1\right) p-2$. Then by Lemma 4.2 , the weight function $\mu(w):=\left|w_{n+1}\right|^{2 s-m p+2}$ belongs to the class $A_{p}$. Fix some $f \in H^{p}\left(\mathbb{B}_{*},|\zeta \bullet \zeta|^{s} d \theta\right)$ and set

$$
g(z):=z_{n+1}^{m} f(F(z)), \quad \forall z \in \mathbb{M} .
$$


Then we obtain $g \in H^{p}\left(\mathbb{M},\left|w_{n+1}\right|^{2+2 s-m p} d \sigma\right)$ by virtue of formula (4.2). From the choice of $m$, it follows that there is an $d \in \mathbb{R}$ such that $1<d<p$ and $(2+2 s-m p) d<2(p-d)$. Therefore, by Hölder's inequality, we obtain, for every $0<r<1$,

$$
\begin{aligned}
& \int_{\partial \mathbb{M}}|g(r w)|^{d} d \sigma(w) \leq\left(\int_{\partial \mathbb{M}}|g(r w)|^{p}\left|w_{n+1}\right|^{2+2 s-m p} d \sigma(w)\right)^{\frac{d}{p}} \\
& \cdot\left(\int_{\partial \mathbb{M}}\left|w_{n+1}\right|^{\frac{-(2+2 s-m p) d}{p-d}} d \sigma(w)\right)^{\frac{p-d}{p}} \\
& \leq C\|g\|_{H^{p}\left(\mathbb{M},\left|w_{n+1}\right|^{2+2 s-m p} d \sigma\right)}^{d}<\infty
\end{aligned}
$$

where the second estimate holds by using Lemma 4.1 .

Thus $g \in H^{d}(\mathbb{M}, \sigma)$. Applying part (i) of Theorem 3.14, we obtain

$$
g=\mathcal{P}_{\mathbb{M}}\left[g^{*}\right]
$$

Next, we first apply the Korányi type inequality (Theorem 3.7), then Theorem 3 of Calderón's work [2] and formula (4.2), and obtain

$$
\begin{aligned}
\int_{\partial \mathbb{M}}\left|M_{\alpha} \mathcal{P}_{\mathbb{M}}\left[g^{*}\right](w)\right|^{p}\left|w_{n+1}\right|^{2+2 s-m p} d \sigma(w) \\
\leq C \cdot \int_{\partial \mathbb{M}}\left|M\left(g^{*}\right)(w)\right|^{p}\left|w_{n+1}\right|^{2+2 s-m p} d \sigma(w) \\
\leq C \cdot \int_{\partial \mathbb{M}}\left|g^{*}(w)\right|^{p}\left|w_{n+1}\right|^{2+2 s-m p} d \sigma(w)<\infty .
\end{aligned}
$$

On the other hand, in view of (4.17) and (4.19), we see that for every $w \in \partial \mathbb{M}$ and $\alpha>2$,

$$
\left|M_{\alpha} \mathcal{P}_{\mathbb{M}}\left[g^{*}\right](w)\right|=\left|M_{\alpha} g(w)\right| \geq C\left|w_{n+1}\right|^{m} \sup _{\frac{1}{2}<r<1}|f(F(r w))| .
$$


Combining the estimates (4.20), (4.21) and formulas (4.12) et (4.17), we obtain

$$
\begin{aligned}
& \int_{\partial \mathbb{M}}\left|M_{\mathrm{rad}}(f \circ F)(w)\right|^{p}\left|w_{n+1}\right|^{2 s+2} d \sigma(w) \\
& \leq C \cdot \int_{\partial \mathbb{M}}\left|(f \circ F)^{*}(w)\right|^{p}\left|w_{n+1}\right|^{2 s+2} d \sigma(w) .
\end{aligned}
$$

Choose $q \in \mathbb{R}$ such that $q>\max \{1, s+2\}$. By Lemma 4.3, we get

$$
\left(M_{\alpha}(f \circ F)(w)\right)^{\frac{p}{q}} \leq C M\left(\left|M_{\mathrm{rad}}(f \circ F)\right|^{\frac{p}{q}}\right)(w), \quad \forall w \in \partial \mathbb{M} .
$$

Since by Lemma 4.2 the weight function $\mu(w):=\left|w_{n+1}\right|^{2 s+2}$ belongs to the class $A_{q}$, using the latter estimate and applying again Theorem 3 of $[\mathbf{2}]$, it follows that

$$
\begin{aligned}
& \int_{\partial \mathbb{M}}\left(M_{\alpha}(f \circ F)(w)\right)^{p}\left|w_{n+1}\right|^{2 s+2} d \sigma(w) \\
\leq & C \cdot \int_{\partial \mathbb{M}}\left[M\left(\left|M_{\text {rad }}(f \circ F)\right|^{\frac{p}{q}}\right)(w)\right]^{q}\left|w_{n+1}\right|^{2 s+2} d \sigma(w) \\
\leq & C \cdot \int_{\partial \mathbb{M}}\left|M_{\text {rad }}(f \circ F)(w)\right|^{p}\left|w_{n+1}\right|^{2 s+2} d \sigma(w) .
\end{aligned}
$$

We deduce from estimates (4.22) and (4.23) that

$$
\begin{array}{rl}
\int_{\partial \mathbb{M}}\left|M_{\alpha}(f \circ F)(w)\right|^{p}\left|w_{n+1}\right|^{2 s+2} & d \sigma(w) \\
\leq C \cdot \int_{\partial \mathbb{M}}\left|(f \circ F)^{*}(w)\right|^{p}\left|w_{n+1}\right|^{2 s+2} d \sigma(w) .
\end{array}
$$

Part (i) of the theorem now follows immediately from (4.2), (4.4) and the latter estimate.

We now turn to prove part (ii). We have already observed in the proof of part (i) of Theorem A that for every $0 \leq r<1$ and $\alpha>2, r \zeta \in D_{\alpha}^{*}(\zeta)$ and the dilated functions $f_{r}$ converge to $f^{*} \theta$-almost every where on $\partial \mathbb{B}_{*}$ as $r \rightarrow 1^{-}$. Hence in view of part (i) of Theorem B and applying the dominated convergence theorem, we see that

$$
\lim _{r \rightarrow 1^{-}}\left\|f^{*}-f_{r}\right\|_{L^{p}\left(\partial \mathbb{B}_{*},|\zeta \bullet \zeta|^{s} d \theta\right)}=0 .
$$


To prove the second equality of part (ii), we remark that

$$
\begin{aligned}
& \lim _{r \rightarrow 1^{-}}\left\|f_{r}\right\|_{L^{p}\left(\partial \mathbb{B}_{*},|\zeta \bullet \zeta|^{s} d \theta\right)}^{p} \\
= & C \lim _{r \rightarrow 1^{-}} \int_{\partial \mathbb{M}}\left|\left(f_{r} \circ F\right)(w)\right|^{p}\left|w_{n+1}\right|^{2 s+2} d \sigma(w) \\
= & C \lim _{r \rightarrow 1^{-}} \int_{\partial \mathbb{M}}\left|w_{n+1}\right|^{2 s+2} d \sigma(w) \frac{1}{2 \pi} \int_{-\pi}^{\pi}\left|\left(f_{r} \circ F\right)\left(e^{i \eta} w\right)\right|^{p} d \eta .
\end{aligned}
$$

Since the function $\left|f_{r} \circ F\right|^{p}$ is subharmonic, we deduce from (4.24) and (4.25) that

$$
\left\|f^{*}\right\|_{L^{p}\left(\partial \mathbb{B}_{*},|\zeta \bullet \zeta|^{s} d \theta\right)}=\lim _{r \rightarrow 1^{-}}\left\|f_{r}\right\|_{L^{p}\left(\partial \mathbb{B}_{*},|\zeta \bullet \zeta|^{s} d \theta\right)}=\|f\|_{H^{p}\left(\mathbb{B}_{*},|\zeta \bullet \zeta|^{s} d \theta\right)} .
$$

This completes the proof of part (ii).

We now arrive at the proof of Theorem $\mathrm{C}$.

Proof of Theorem $C$ : We remark that if $-2<s<\frac{3 p}{2}-2$ and $f \in$ $L^{p}\left(\partial \mathbb{B}_{*},|\zeta \bullet \zeta|^{s} d \theta\right)$, then by virtue of formula (4.2) and Lemma 4.1 we have that,

$$
\int_{\partial \mathbb{B}_{*}}|\zeta \bullet \zeta|^{s} d \theta(\zeta)=\frac{1}{C_{3}} \int_{\partial \mathbb{M}}\left|w_{n+1}\right|^{2 s+2} d \sigma(w)<\infty,
$$

and

$$
\begin{aligned}
& \int_{\partial \mathbb{M}}|T f| d \sigma \leq\left(\int_{\partial \mathbb{M}}\left|w_{n+1}\right|^{\frac{p-(2 s+2)}{p-1}} d \sigma(w)\right)^{\frac{p-1}{p}} \\
& \cdot\left(\int_{\partial \mathbb{M}}|(f \circ F)(w)|^{p}\left|w_{n+1}\right|^{2 s+2} d \sigma(w)\right)^{\frac{1}{p}} \\
& \leq C\|f\|_{L^{p}\left(\partial \mathbb{B}_{*},|\zeta \bullet \zeta|^{s} d \theta\right)}
\end{aligned}
$$

where the second estimate follows from applying Hölder's inequality. Therefore, equation (4.3) holds for every $f \in L^{p}\left(\partial \mathbb{B}_{*},|\zeta \bullet \zeta|^{s} d \theta\right)$ with $-2<s<\frac{3 p}{2}-2$. 
For every $0<r<1$, consider the integral operator $f \longrightarrow \mathcal{S}_{r}[f]$ given by

$$
\mathcal{S}_{r}[f](\zeta):=\int_{\partial \mathbb{M}} S_{\mathbb{M}}(r \zeta, w) f(w) d \sigma(w), \quad \forall \zeta \in \partial \mathbb{M}, \forall f \in L^{1}(\partial \mathbb{M}, \sigma) .
$$

Observe that by virtue of estimate (3.12) and Theorem 3.15, the integral operator $\mathcal{S}_{r}$ is a singular integral in the space of homogeneous type ( $\partial \mathbb{M}, d, \sigma)$. Consequently, we may apply the weighted theory of singular integral in $[3]$ to $\mathcal{S}_{r}$. From the hypothesis on $s$ and by Lemma 4.2, the weight function $\mu(w):=\left|w_{n+1}\right|^{2 s-p+2}$ belongs to the class $A_{p}$. Then it follows from $[\mathbf{3}]$ that

$$
\begin{aligned}
\sup _{0<r<1} \int_{\partial \mathbb{M}}\left|\mathcal{S}_{\mathbb{M}}[T f](r w)\right|^{p}\left|w_{n+1}\right|^{2 s-p+2} d \sigma(w) \\
\leq C \int_{\partial \mathbb{M}}|T f(w)|^{p}\left|w_{n+1}\right|^{2 s-p+2} d \sigma(w),
\end{aligned}
$$

for every $f \in L^{p}\left(\partial \mathbb{B}_{*},|\zeta \bullet \zeta|^{s} d \theta\right)$.

Using (4.1)-(4.3) and the remark made at the beginning of our proof, we obtain

$$
\begin{aligned}
\int_{\partial \mathbb{M}}\left|\mathcal{S}_{\mathbb{M}}[T f](r w)\right|^{p}\left|w_{n+1}\right|^{2 s-p+2} d \sigma(w) & \\
& =C r^{p} \int_{\partial \mathbb{B}_{*}}\left|\mathcal{S}_{\mathbb{B}_{*}}[f](r \zeta)\right|^{p}|\zeta \bullet \zeta|^{s} d \theta(\zeta),
\end{aligned}
$$

and

$$
\int_{\partial \mathbb{M}}|T f(w)|^{p}\left|w_{n+1}\right|^{2 s-p+2} d \sigma(w)=C \int_{\partial \mathbb{B}_{*}}|f(\zeta)|^{p}|\zeta \bullet \zeta|^{s} d \theta(\zeta) .
$$

Combining (4.26)-(4.28), we get

$$
\text { (4.29) } \sup _{\frac{1}{2}<r<1} \int_{\partial \mathbb{B}_{*}}\left|\mathcal{S}_{\mathbb{B}_{*}}[f](r \zeta)\right|^{p}|\zeta \bullet \zeta|^{s} d \theta(\zeta) \leq C \int_{\partial \mathbb{B}_{*}}|f(\zeta)|^{p}|\zeta \bullet \zeta|^{s} d \theta(\zeta) .
$$

Consider the function $g \in H\left(\mathbb{B}_{*}\right)$ given by $g:=\mathcal{S}_{\mathbb{B}_{*}}[f]$. By virtue of (4.29), $g$ is in $H^{p}\left(\mathbb{B}_{*},|\zeta \bullet \zeta|^{s} d \theta\right)$. Therefore, Theorem B, applied to $g$, gives that

$$
\int_{\partial \mathbb{B}_{*}}\left|M_{\alpha} g(\zeta)\right|^{p}|\zeta \bullet \zeta|^{s} d \theta(\zeta) \leq C \sup _{\frac{1}{2}<r<1} \int_{\partial \mathbb{B}_{*}}|g(r \zeta)|^{p}|\zeta \bullet \zeta|^{s} d \theta(\zeta) .
$$


Combining (4.29) and (4.30), we obtain the desired conclusion of Theorem C.

Acknowledgement. The author would like to thank E. H. Youssfi for his recommandations and encouragement.

\section{References}

[1] P. Ahern And J. Bruna, Maximal and area integral characterizations of Hardy-Sobolev spaces in the unit ball of $\mathbb{C}^{n}$, Rev. Mat. Iberoamericana 4(1) (1988), 123-153.

[2] A.-P. CALderón, Inequalities for the maximal function relative to a metric, Studia Math. 57(3) (1976), 297-306.

[3] R. R. Coifman and C. Fefferman, Weighted norm inequalities for maximal functions and singular integrals, Studia Math. 51 (1974), 241-250.

[4] K. T. Hahn And P. Pflug, On a minimal complex norm that extends the real Euclidean norm, Monatsh. Math. 105(2) (1988), $107-112$.

[5] K-T. KIM, Automorphism groups of certain domains in $\mathbb{C}^{n}$ with a singular boundary, Pacific J. Math. 151(1) (1991), 57-64.

[6] A. KorÁnyi, Harmonic functions on Hermitian hyperbolic space, Trans. Amer. Math. Soc. 135 (1969), 507-516.

[7] G. Mengotti, Duality theorems for certain analytic spaces on the minimal ball, Arch. Math. (Basel) 75(5) (2000), 389-394.

[8] G. Mengotti And E. H. Youssfi, The weighted Bergman projection and related theory on the minimal ball, Bull. Sci. Math. 123(7) (1999), 501-525.

[9] A. Nagel And E. M. Stein, On certain maximal functions and approach regions, Adv. in Math. 54(1) (1984), 83-106.

[10] A. Nagel, E. M. Stein And S. WAinger, Boundary behavior of functions holomorphic in domains of finite type, Proc. Nat. Acad. Sci. U.S.A. 78(11), part 1, (1981), 6596-6599.

[11] A. Nagel, E. M. Stein and S. Wainger, Balls and metrics defined by vector fields. I. Basic properties, Acta Math. 155(1-2) (1985), 103-147.

[12] K. Oelueklaus, P. Pflug and E. H. Youssfi, The Bergman kernel of the minimal ball and applications, Ann. Inst. Fourier (Grenoble) 47(3) (1997), 915-928.

[13] K. Oelueklaus and E. H. Youssfi, Proper holomorphic mappings and related automorphism groups, J. Geom. Anal. 7(4) (1997), 623-636. 
[14] N. Ourimi, Applications holomorphes propres entre certains domaines bornés de $\mathbb{C}^{n}, C$. R. Acad. Sci. Paris Sér. I Math. 326(9) (1998), 1063-1068.

[15] P. Pflug And E. H. Youssfi, The Lu Qi-Keng conjecture fails for strongly convex algebraic domains, Arch. Math. (Basel) 71(3) (1998), 240-245.

[16] W. Rudin, "Function theory in the unit ball of $\mathbb{C}^{n}$ ", Grundlehren der Mathematischen Wissenschaften 241, Springer-Verlag, New York-Berlin, 1980.

[17] E. M. STEIn, "Boundary behavior of holomorphic functions of several complex variables", Mathematical Notes 11, Princeton University Press, Princeton, N.J., University of Tokyo Press, Tokyo, 1972.

[18] N. Viêt ANH AND E. H. Youssfi, Lipschitz estimates for the $\bar{\partial}$-equation on the minimal ball, Michigan Math. J. 49(2) (2001), 299-323.

[19] E. H. Youssfi, Proper holomorphic lifting of the Bergman and Szegö kernels for some complex domains, Preprint (1998).

Université de Paris-Sud 11

Bâtiment 425-Mathématiques

F-91405 Orsay Cedex

France

E-mail address: Viet-Anh.Nguyen@math.u-psud.fr

Rebut el 13 de febrer de 2001. 